\title{
User activity recognition system to improve the performance of environmental control interfaces: a pilot study with patients
}

\author{
Arturo Bertomeu-Motos ${ }^{1 *}$ (D), Santiago Ezquerro ${ }^{1}$, Juan A. Barios ${ }^{1}$, Luis D. Lledó ${ }^{1}$, Sergio Domingo², \\ Marius Nann ${ }^{4}$, Suzanne Martin ${ }^{3}$, Surjo R. Soekadar ${ }^{5}$ and Nicolas Garcia-Aracil ${ }^{1}$
}

\begin{abstract}
Background: Assistive technologies aim to increase quality of life, reduce dependence on care giver and on the long term care system. Several studies have demonstrated the effectiveness in the use of assistive technology for environment control and communication systems. The progress of brain-computer interfaces (BCI) research together with exoskeleton enable a person with motor impairment to interact with new elements in the environment. This paper aims to evaluate the environment control interface (ECI) developed under the AIDE project conditions, a multimodal interface able to analyze and extract relevant information from the environments as well as from the identification of residual abilities, behaviors, and intentions of the user.
\end{abstract}

Methods: This study evaluated the $\mathrm{ECl}$ in a simulated scenario using a two screen layout: one with the $\mathrm{ECl}$ and the other with a simulated home environment, developed for this purpose. The sensorimotor rhythms and the horizontal oculoversion, acquired through $\mathrm{BCI} 2000$, a multipurpose standard BCl platform, were used to online control the $\mathrm{ECl}$ after the user training and system calibration. Eight subjects with different neurological diseases and spinal cord injury participated in this study. The subjects performed simulated activities of daily living (ADLs), i.e. actions in the simulated environment as drink, switch on a lamp or raise the bed head, during ten minutes in two different modes, AIDE mode, using a prediction model, to recognize the user intention facilitating the scan, and Manual mode, without a prediction model.

Results: The results show that the mean task time spent in the AIDE mode was less than in the Manual, i.e the users were able to perform more tasks in the AIDE mode during the same time. The results showed a statistically significant differences with $p<0.001$. Regarding the steps, i.e the number of abstraction levels crossed in the ECI to perform an $A D L$, the users performed one step in the $90 \%$ of the tasks using the AIDE mode and three steps, at least, were necessary in the Manual mode. The user's intention prediction was performed through conditional random fields (CRF), with a global accuracy about $87 \%$.

Conclusions: The environment analysis and the identification of the user's behaviors can be used to predict the user intention opening a new paradigm in the design of the ECls. Although the developed ECI was tested only in a simulated home environment, it can be easily adapted to a real environment increasing the user independence at home.

Keywords: Environment control interface, Brain-computer interface, User intention prediction, Multimodal system, Brain injury, Spinal-cord injury

*Correspondence: abertomeu@umh.es

${ }^{1}$ Miguel Hernández University of Elche, Av. Universidad w/n, Ed. Innova, 03202

Elche, Spain

Full list of author information is available at the end of the article

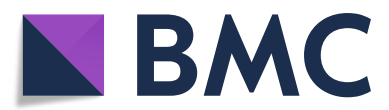

(C) The Author(s). 2019 Open Access This article is distributed under the terms of the Creative Commons Attribution 4.0 International License (http://creativecommons.org/licenses/by/4.0/), which permits unrestricted use, distribution, and reproduction in any medium, provided you give appropriate credit to the original author(s) and the source, provide a link to the Creative Commons license, and indicate if changes were made. The Creative Commons Public Domain Dedication waiver (http://creativecommons.org/publicdomain/zero/1.0/) applies to the data made available in this article, unless otherwise stated. 


\section{Background}

It is estimated that one in six people in the world are diagnosed with a neurological disorder and this number is expected to rise considerable due to extensions of life expectancy [1]. A neurological condition is a damage to the brain, spinal column or nerves due to illness or injury such as spinal cord injury, acquired brain damage, stroke, motor neurons disease and locked in syndrome. Neurological disorders are considered the primary cause of disability in modern society $[1,2]$. The debilitating consequences of neurological disorders include communication difficulties, impaired memory, inappropriate behavior, physical disability, restricted independence, social isolation and poor quality of life.

Assistive technologies aim to increase quality of life [3-6], reduce dependence on care giver [7] and reduce dependence on the long term care system [8]. Several studies have demonstrated the effectiveness in the use of environment control interfaces (ECI) for environment control or communication through voice commands [9], scan interfaces based on grid structure, eye tracking [10-12] or brain-computer interface (BCI) based on P300 [13], among others. These software platforms actively aid during the Activities of Daily Living (ADL) improving the independence both at home and outside. However, these platforms are based in a manual scan over the different abstraction levels of the ECIs and the scan speed only depends on the users familiarization with the system and the configuration of the grids over the different menus. Thus, introducing the user environment and behavior into this loop will help the navigation agility in the ECIs.

On the other hand, The progress of BCI research together with exoskeleton enables a person with motor impairment to interact with new elements of the environment $[14,15]$. Thus, this progress will deliver new scenarios to $\mathrm{BCI}$ systems out of laboratories and move $\mathrm{BCI}$ into the domestic environment. The AIDE project ${ }^{1}$ aims to create new shared-control paradigm for assistive devices that integrates information from identification of residual abilities, behaviors, emotional state and intentions of the user on one hand and analysis of the environment and context factors on the other hand. In this context, a hybrid BCI model was chosen to control the ECI. It was developed as a fusion between non-invasive electroencephalography (EEG) and electrooculography (EOG) system [16]. The EEG records the sensorimotor rhythms (SMR) called event-related desynchronization (ERD) and event-related synchronization (ERS) during a motor imagery (MI) task [17] whilst the EOG records the horizontal oculoVersion (HOV).

This paper aims to evaluate the ECI developed under the AIDE project conditions, a multimodal system developed to assist people with acquired brain damage or neurodegenerative diseases that need a wheelchair and has low or any upper limbs mobility in their ADLs, in a simulated environment able to detect the user intention through the environment analysis and the identification of the user's behaviors, based on a conditional random fields (CRF) model [18]. Thus, the handling of the interface was studied in two different ways, with and without the prediction of the user's intention. Users with neurological and muscular diseases and spinal cord injury (SCI) tested the system on a virtual home due to the early phase of the project.

\section{Methods}

This study evaluated the ECI in a simulated scenario under the AIDE project conditions, a multimodal interface able to analyze and extract relevant information from the environments as well as from the identification of residual abilities, behaviors, and intentions of the user. It consisted in a two screen layout: ECI and simulated room, with an EEG and EOG data acquisition system (see Fig. 1).

\section{Environment control interface}

The environment control system used in this experimentation was based in two main software components: GRID3 from Smartbox ${ }^{2}$, a commercial augmentative and alternative communication (AAC) solution, and SHX, a specific developed software, presented in Fig 2. The ECI had three different abstraction levels: 1) related with the room (room menu), 2) related to the activities that can be performed in a specific room (activity menu), and 3) related to the actions regarding a specific activity (ADL menu). The jump between two consecutive abstraction levels will be named as step.

Levels one and two were specific grid sets, created in GRID3, to be used in the context of the experimentation. They include grids for the different rooms, communication, control a wheelchair and control an exoskeleton arm. In all grids, a color code has been used: red for the alarm; green for communication, computer control and digital leisure; white for wheelchair and arm control (not used in this experimentation); light blue for the rooms; dark blue for environmental control activity. The dark blue cells are referred to environmental control activities and linked Grid3 with the SHX application. The user could scan across the different cells, select one, and then confirm or cancel the selection using the chosen signals (EOG, EEG, eye tracking, etc.).

SHX is a custom build solution for environmental control management, level three. It allowed the user to easily configure and select the actions programmed for a specific activity. Different scenes for each of the possible activities in every room were created. Here too, the user could scan across the different cells, select one, and then confirm or cancel the selection using the chosen signals (EOG, EEG, eye tracking, etc.). 


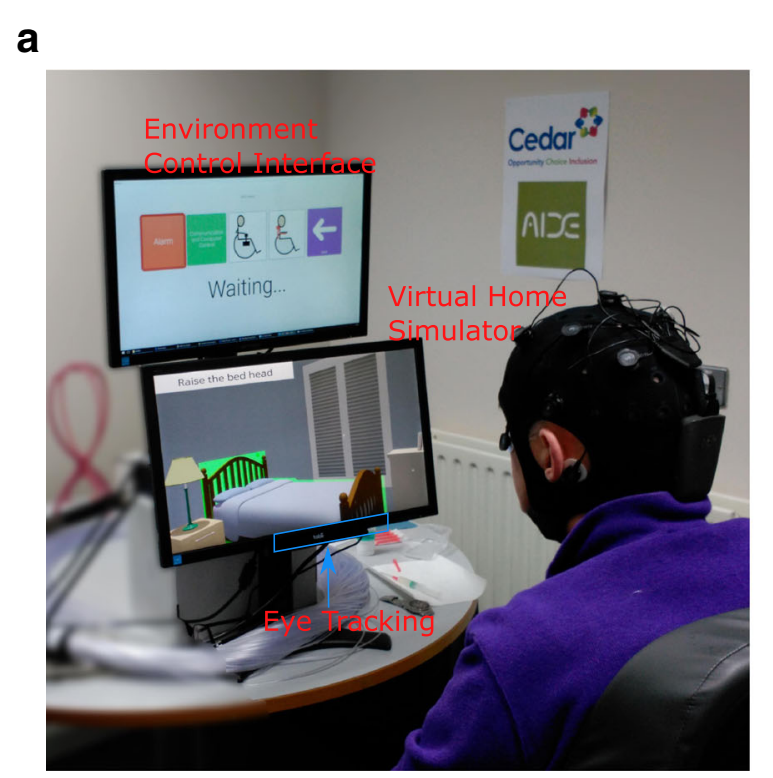

\section{b}

Fig. 1 Experimentation setup. The experimental setup was composed by two screens layout: ECI \& Virtual home simulator, and an eye tracking placed in the bottom screen. The users performed tasks in the simulated environment (a) and they also interacted with real elements (b)

\section{Data acquisition}

The acquisition of the brain activity was performed with eight solid-gel electrodes placed according to international 10-20 system placed at F3, C3, Cz, P3, T7 and Mastoid, a reference electrode was placed on $\mathrm{C} 4$ and the ground on FpZ. Furthermore, two electrodes were placed on the outer canthus of the eyes to the EOG signal recording. The EEG/EOG signal was acquired via Bluetooth through the Neuroelectrics amplifier (Enobio, Neuroelectrics, Barcelona, Spain). Skin/electrode resistance was kept below $12 \mathrm{kOhm}$. a

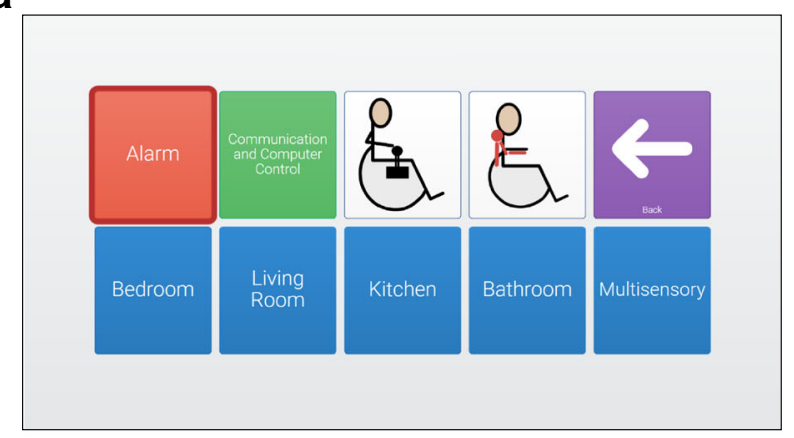

b

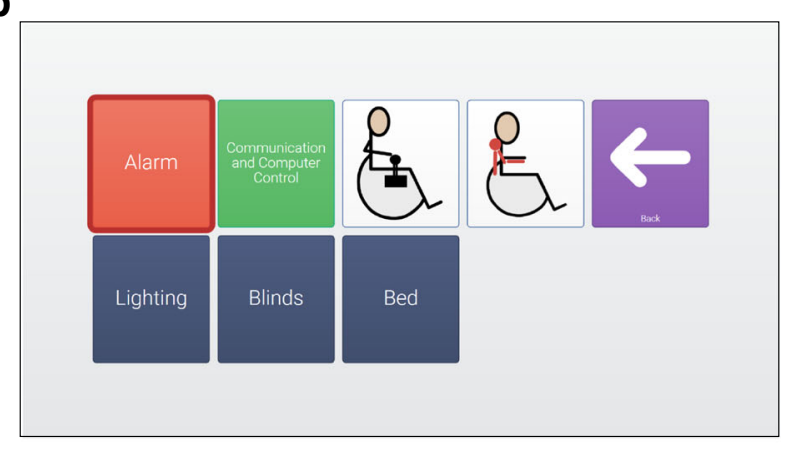

C

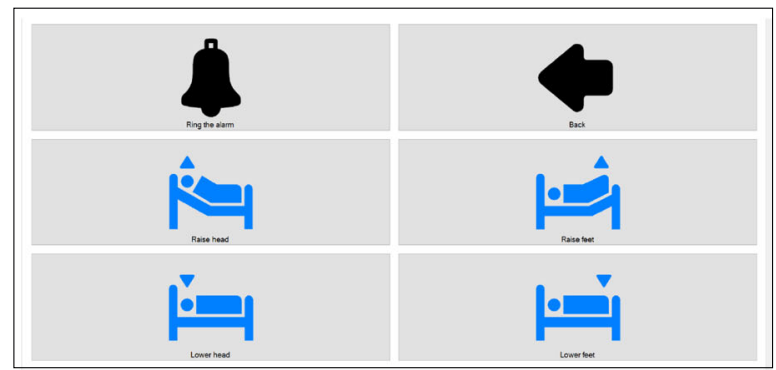

Fig. 2 Environment Control Interface. The ECI had three different abstraction levels: 1) related with the room, 2) related to the activities that can be performed in a specific room and 3) related to the actions regarding a specific activity. An example menu of each level is shown in (a), (b) and (c) respectively 
A real-time SMR-based BCI was implemented using BCI2000, a freely distributed software for multipurpose standard BCI platform [19]. EEG and EOG were recorded at a sampling rate of $500 \mathrm{~Hz}$, bandpass filtered at 0.4$70 \mathrm{~Hz}$ and pre-processed using a small Laplacian filter. Based on the maximum values for basal ERD, the ongoing EEG signal associated with the specified SMR rhythm frequency range (11-14 Hz) calculated from C3 electrode, a subject's individual motor imagery discrimination threshold were set. The EOG discrimination thresholds were calculated regarding the average amplitudes of horizontal saccades. These individual parameters were obtained from the training session and used for later online $\mathrm{BCI}$ control [20].

\section{Prediction model}

The proposed ECI combines the environmental information and context factors together with user's behaviors in order to detect the user intention. Thus, the input information of the prediction model was a sequence of data, the user is moving and looking at the environment, that had to be labeled. In this context, different models were tested (time-delay neural networks, decision trees, hidden markov model (HMM)...) and the CRF model was chosen, showing the best results. The CRF model is a probabilistic model for segmentation and labeling sequence data. This discriminate model takes into account not only the current state but also the previous states to perform its prediction. A conditional model specifies the probabilities of possible label sequences given an observation sequence in contras of the generative models that make very strict independence assumptions on the observation, for instance conditional independence given the labels as HMM [21].

In our case, the inputs of the system were: localization, objects in the environment, object that the user is looking at, temperature of the room, brightness of the room and day time; the output was the ADL menu, i.e. the most probable action that the user wanted to perform that were directly linked with a specific ADL (see Table 1.

\section{Participants}

Eight persons with different neurological pathology and spinal cord injury participated in this study $(37 \pm 15$ years old), their demographic and clinical characteristics are listed in (Table 2). The subjects were evaluated before the experiment with the barthel index [22]. All participants gave informed consent using their standard communication channel prior to participation in the study. The protocol was approved by the Office Research Ethics Northern Ireland - approval granted for project (15/NE/0384).
Table 1 Correlation between the ADLs and ADL menu name, in the third abstraction level

\begin{tabular}{|c|c|c|c|}
\hline ADLs & ADL menu & ADLs & ADL menu \\
\hline $\begin{array}{l}\text { Open/close } \\
\text { fridge }\end{array}$ & Drink or eat & $\begin{array}{l}\text { Switch on/off air } \\
\text { conditioner }\end{array}$ & Air conditioner \\
\hline $\begin{array}{l}\text { Open/close } \\
\text { microwave }\end{array}$ & Drink or eat & Brushing teeth & Teeth \\
\hline Eating task & Eat & Washing face & Face \\
\hline Drinking task & Drink & $\begin{array}{l}\text { Raise/lower the } \\
\text { bed head }\end{array}$ & Bed \\
\hline $\begin{array}{l}\text { Switch on/off } \\
\text { Music }\end{array}$ & Entertainment & $\begin{array}{l}\text { Raise/lower the } \\
\text { bed feet }\end{array}$ & Bed \\
\hline Switch on/off PC & Entertainment & $\begin{array}{l}\text { Open/close the } \\
\text { blinds }\end{array}$ & Blinds \\
\hline Switch on/off TV & TV & $\begin{array}{l}\text { Switch on/off the } \\
\text { light }\end{array}$ & Light \\
\hline
\end{tabular}

\section{Experimental protocol}

Subjects were sitting in his/her own wheelchair in front of a table with two screens, as shown in Fig. 1. The screens were used to show the ECI and the virtual home simulator. Subjects used the AIDE multimodal interface, hybrid EEG EOG system, to online control the ECI and preform specific ADLs. Two modes were tested:

A) MANUAL mode: the user had to navigate through the three abstraction levels in order to accomplish the task showed in the virtual house. The objects related with the corresponding task were surrounded by a green color in the virtual house environment and the task appeared in the right top corner.

B) AIDE mode: in this mode the prediction model was used. The user had to look at the objects related to the specific ADL, showed like in the other mode, and, after the user's intention prediction, the ECI directly jumped to the corresponding ADL menu. Then the user had to navigate like MANUAL mode. In case of

Table 2 Demographic and clinical characteristics of participants

\begin{tabular}{lllll}
\hline ID Patient & Sex & Age & Diagnosis & Barthel score \\
\hline 1 & Male & 32 & C4 SCl & $4 / 20$ \\
2 & Male & 22 & $\begin{array}{l}\text { Duchenne } \\
\text { Muscular } \\
\text { Dystrophy }\end{array}$ & $6 / 20$ \\
3 & Male & 55 & $\begin{array}{l}\text { Brain stem } \\
\text { strokes }\end{array}$ & $16.5 / 20$ \\
4 & Male & 30 & C4/C5 SCl & $2 / 20$ \\
5 & Female & 20 & C6/C7 SCl & $10 / 20$ \\
6 & Male & 58 & Ischemic Stroke & $19 / 20$ \\
7 & Male & 55 & $\begin{array}{l}\text { Multiple Systems } \\
\text { Atrophy }\end{array}$ & $5 / 20$ \\
\hline & Male & 30 & C6/C7 SCl & $9 / 10$ \\
\hline
\end{tabular}


wrong prediction, the user had to manually go back, the second abstraction level, and complete the corresponding ADL. The observed objects were online detected from the eye tracking Tobbi ${ }^{3}$ PCEye go, placed on the virtual environment screen, and the rest of the inputs were online simulated.

Each subject performed two experimental sessions in two consecutive days. The first session was for training and calibration purpose as well as for the familiarization with the systems to be controlled. This session lasted around $60-80 \mathrm{~min}$. In the last part of this session the user learned how to use the hybrid EEG/EOG interface in order to control the ECI. An example of MI and EOG movements in the training session are shown in Fig. 3c and $d$, respectively.

The second session lasted a maximum of $60 \mathrm{~min}$. The setup and familiarization phase took approximately 15 min (subjects have already tested the system in the first session). They had 10 min to perform a predefined ADLs list in both AIDE and Manual modes (all ADLs can be observed in Table 1). Each ADL can be a single action, it has a visual effect on the house simulator, e.g. swith on a lamp, or an exoskeleton action, the simulator play a short video showing the corresponding action, e.g. drink from a glass. The order of the modes was randomly selected and, before each mode, a baseline of 3 min was acquired. During the break ( $5 \mathrm{~min}$ ) and at the end of this session the subjects answered the NASA task load index (tlx) questionnaire [23].

The scan in the ECI was performed through lookingright eye movements (Fig. 3b) implying a forward displacement of the grid marker (Fig. 3a-e). Once the subject stopped at one grid a customized time, chosen in the first session, the ECI 'switch off' the rest of the grids (Fig. 3f). Then, the next level or the action in the ECI was achieved by on-line ERD detection, like the subjects learned during the first session (Fig. 3a). On the other hand, if the user did not want to click on this specific grid, in the phase Fig. 3f, a looking-left eye movement returns the ECI to the phase Fig. 3a. When the user performs an action, a visual feedback is presented in the virtual home regarding to the action performed (Fig. $3 \mathrm{~g}$ ) and he/she waited for the next task.

After both modes, they were instructed to interact with real elements through the ECI and watch a video using objects of a multi-sensory room, as can be observed in Fig. 1b.

\section{Results}

The users performed simulated ADLs during $10 \mathrm{~min}$ in a virtual home using an ECI in both Manual and $A I D E$ modes. The number of the performed tasks with respect to the mean time spent per user is presented in Fig. 4a. Furthermore, it has been trained a Support Vector Machine (SVM) model with Gaussian kernel to estimate the boundary between both modes (yellow line in Fig. 4a). It should be noted that statistically significant differences between both modes in terms of number of tasks and mean tasks time is shown ( $p-$ value $<0.001$ using Wilcoxon test). The steps distribution that the users performed in both modes are shown in Fig. 4c. ADLs manually omitted tasks were excluded from the study due to the subject was blocked during the ECI scan caused by frustration or fatigue over a specific task.

On the other hand, the AIDE mode uses a CRF model, previously trained with simulated data using the same virtual home, to predict the user intention. Thus, the confusion matrix of the prediction model regarding the ADL menus is presented in Fig. 4b. In addition, the results obtained through the NASA tlx questionnaire are presented in Fig. 5.

\section{Discussion}

The AIDE project aims to develop a multimodal system in order to help people with neurological diseases wearing a wheelchair. The presented environment allowed the user to navigate through a virtual house and perform several ADLs using a developed ECI. Two modes were estudied, the AIDE mode, that used a CRF model to predict the user intention and ease the ECI scan, and the Manual mode, that needed a complete scan through the ECI to perform a specific ADL. The ECI was online controlled using the AIDE multimodal system based on a combination of EEG and EOG wireless acquisition system [17].

The results presented in Fig. 4a show the mean time per task spent in the AIDE mode is less than in the Manual mode being able to perform more tasks in the same time, both modes show statistically significant differences $(p<0.001)$. Furthermore, both modes can be easily classified by training a SVM model with Gaussian kernel, the boundary is presented with a yellow line in Fig. 4a.

On the other hand, Fig. 4c shows the difference between both groups in terms of steps, i.e. the ECI abstraction levels that the user had to cross in order to perform a specific ADL. It must be noticed that in the AIDE mode, the users performed one step in the $90 \%$ of the tasks and three steps, at least, were necessary in the Manual mode. Regarding the $A I D E$ mode, three steps were necessary only when the CRF model realized a wrong prediction and, therefore, the user had to return to the activity menu and select the proper ADL menu. Although a bad prediction is sometimes performed, the multimodal system helps in terms of location, i.e. the activity that the system predicts is always related with the room where the user is, facilitating the navigation. Perform five or seven steps in the Manual mode implies that a wrong abstraction level was selected, 


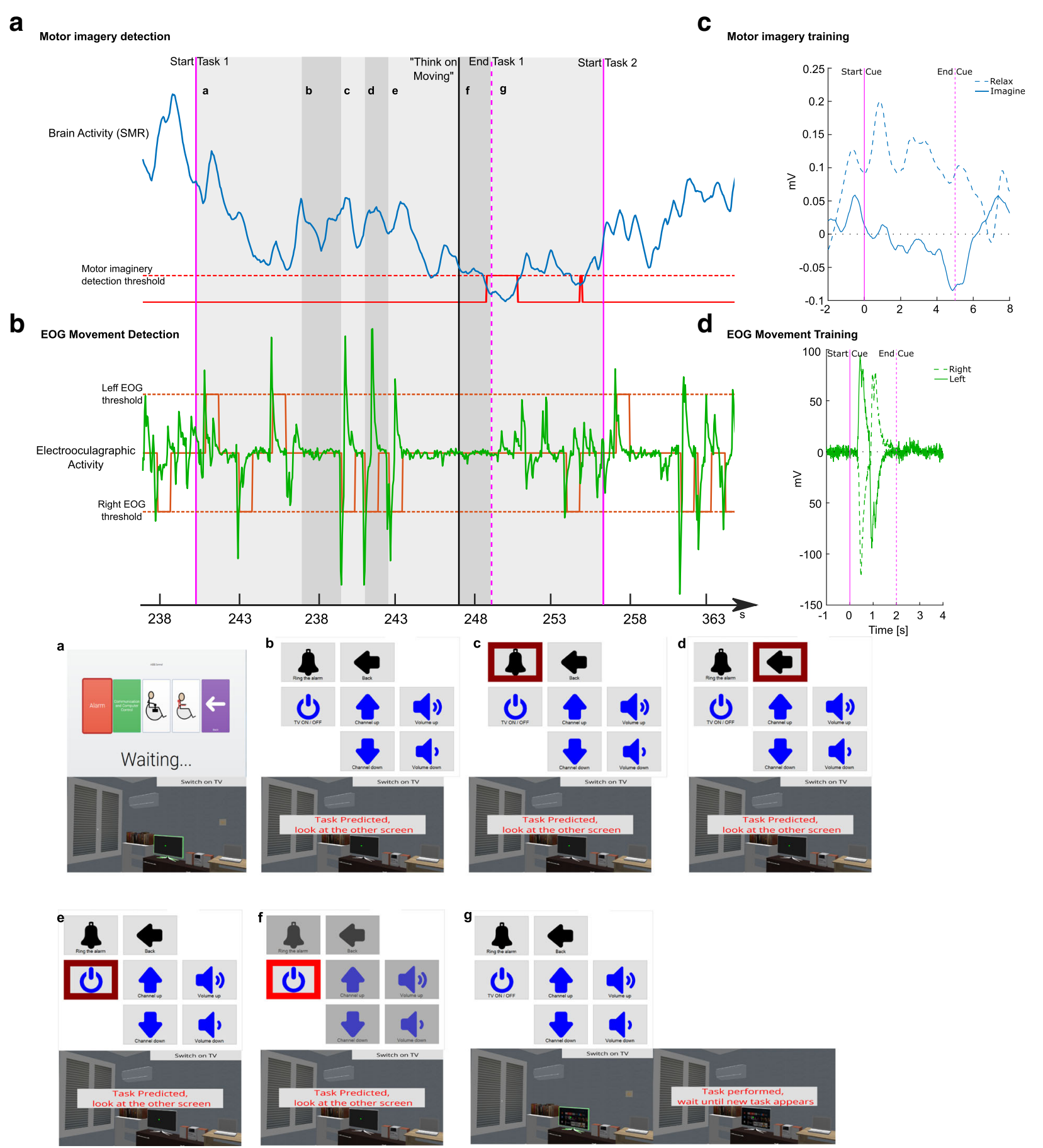

Fig. 3 Multimodal system processing for one ADL in AIDE mode. The user had to perform different actions in order to execute the corresponding ADLs, in this example, the user had to switch on the TV, phases a-g show the behavior of both screens during the task. EEG (a) and EOG (b) signals were acquired to online control the ECI in order to perform ADLs in a virtual house. When the task started (vertical purple line), the scan through the $\mathrm{ECI}$ was performed by EOG activity detection [orange line in (b)], i.e. when HOV activity exceeded the threshold [indicated by the orange dashed line in (b)] the grid marker moved forward (phases a-e). Once the subject stopped at one grid, a task confirmation was needed [indicated by the vertical black line] and the ECI 'switched off' the rest of the grids indicating this purpose (phase f). The confirmation was performed by the detection of SMR-ERD [indicated by red line in (a)] and the action was done, so the ADL finished (vertical dotted purple line). This ADL was performed in one step, i.e. the user only needed to navigate through the last abstraction level to complete the task. Before the experimentation, the user was trained in motor imagery (c) and EOG movement (d) to the set up the control system with the personalized parameters 


\section{a}

Number of the tasks and mean time spent per user

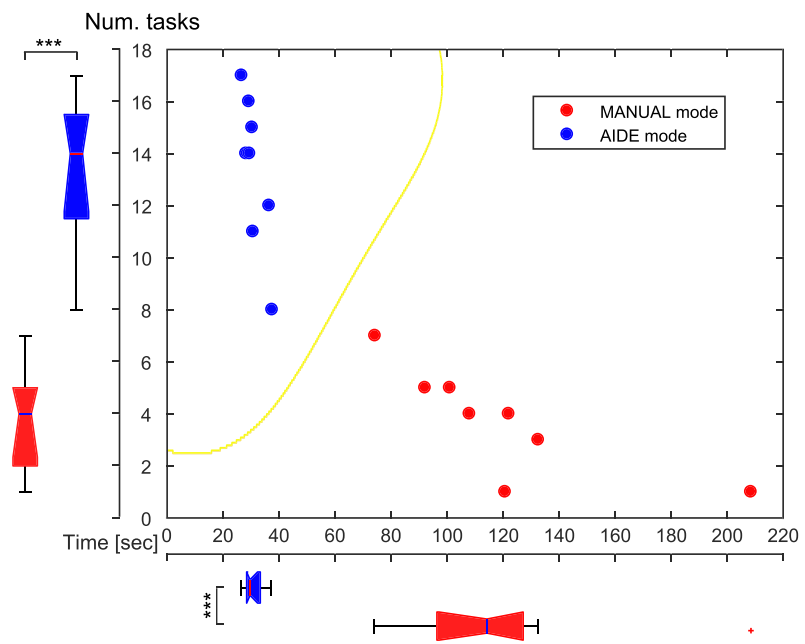

b

Confusion matrix of the prediction model

\begin{tabular}{|c|c|c|c|c|c|c|c|c|c|c|c|}
\hline Drink & 0.4706 & 0 & 0 & 0 & 0 & 0 & 0 & 0 & 0 & 0 & 0 \\
\hline Eat & 0.5294 & 1 & 0 & 0 & 0 & 0 & 0 & 0 & 0 & 0 & 0 \\
\hline Air conditioner & 0 & 0 & 0.8571 & 0 & 0 & 0 & 0 & 0 & 0 & 0 & 0 \\
\hline TV & 0 & 0 & 0 & 0.7143 & 0 & 0 & 0 & 0 & 0 & 0 & 0 \\
\hline Entertainment & 0 & 0 & 0.1429 & 0.2857 & 0 & 0 & 0 & 0 & 0 & 0 & 0 \\
\hline Blinds & 0 & 0 & 0 & 0 & 0 & 1 & 0 & 0 & 0 & 0 & 0 \\
\hline Light & 0 & 0 & 0 & 0 & 0 & 0 & 1 & 0 & 0.1 & 0 & 0 \\
\hline Teeth & 0 & 0 & 0 & 0 & 0 & 0 & 0 & 0.8 & 0 & 0 & 0 \\
\hline Face & 0 & 0 & 0 & 0 & 0 & 0 & 0 & 0.2 & 0.9 & 0 & 0 \\
\hline Bed & 0 & 0 & 0 & 0 & 0 & 0 & 0 & 0 & 0 & 1 & 0 \\
\hline Accuracy & 0 & 0 & 0 & 0 & 0 & 0 & 0 & 0 & 0 & 0 & 0.8692 \\
\hline
\end{tabular}

\begin{tabular}{ccccc}
\hline ID User & \multicolumn{2}{c}{ Number of Tasks } & \multicolumn{2}{c}{ Mean time [s] } \\
\hline & AIDE & MANUAL & AIDE & MANUAL \\
\hline 1 & 16 & 1 & 29.2 & 120.7 \\
2 & 14 & 1 & 28.2 & 208.5 \\
3 & 11 & 5 & 30.6 & 100.9 \\
4 & 14 & 5 & 29.4 & 92.1 \\
5 & 17 & 7 & 26.6 & 74.3 \\
6 & 12 & 3 & 36.5 & 132.6 \\
7 & 8 & 4 & 37.5 & 108.1 \\
8 & 15 & 4 & 30.4 & 122.1 \\
\hline \multicolumn{4}{c}{ p-value $<0.001$} & \multicolumn{2}{c}{ p-value $<0.001$} \\
\hline
\end{tabular}

C

Steps distribution regarding all the tasks

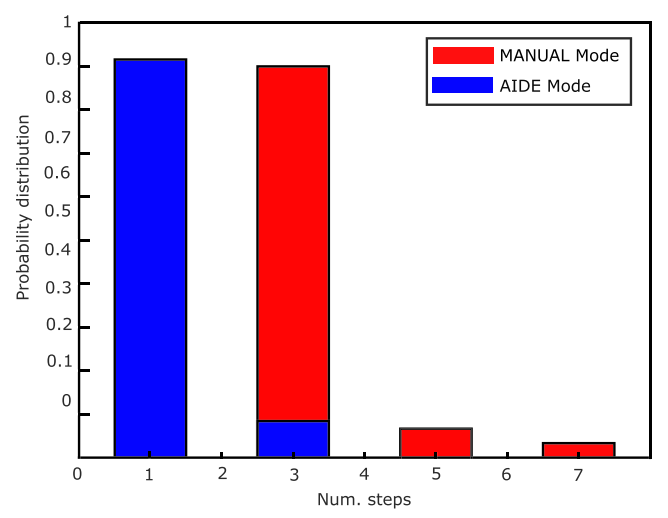

Fig. 4 Environment control interface performance. The users performed two different trials with the same goal: complete as many tasks as possible in 10 min by using the Manual mode and the AIDE mode (a). Both modes have been used to train a SVM model with Gaussian kernel and find the boundary between them [yellow line in (a)]. The interaction with the ECI was measured by steps that a user had to perform in order to complete the tasks (c). Furthermore, in AIDE mode the user's intention prediction was performed through CRF model and, therefore, the confusion matrix of the model is obtained (b)

due to user confusion or lack of practice, and the user had to go back in the ECI.

The AIDE mode uses a CRF model to perform the predictions about the user's intention. The model uses the information of the virtual home and the object that the user is looking at, acquired thought an eye tracking device. The model was previously trained with simulated data using the same virtual house environment. Thus, Fig. $4 \mathrm{~b}$ shows the confusion matrix of the prediction model, regarding the ADL menu, with a global accuracy about $87 \%$. The CRF model, as it takes into account not only the current state but also the previous states to perform its prediction, it could fail in the prediction of task with common features. Therefore, the ADLs related to the Drink menu are sometimes predicted as the ADLs related with the Eat menu, in this case around the $50 \%$ of the trials. In addition, $T V$ and the Teeth menus are rarely selected as Entertainment and Face menu, respectively, by the prediction model.

After each mode, the subjects answered the NASA tlx questionnaire in order to assess the workload between the modes, showed in Fig. 5. Unexpectedly, it has not statistically significant differences, so we can say that the users do not notice subjective differences between both modes in terms of workload. It can be explained because it was the first time that the users handle the complex multimodal control system (EEG+EOG) with this ECI. We assume that, observing the results presented in Fig 4, the workload should decrease, at least, in the AIDE mode. 


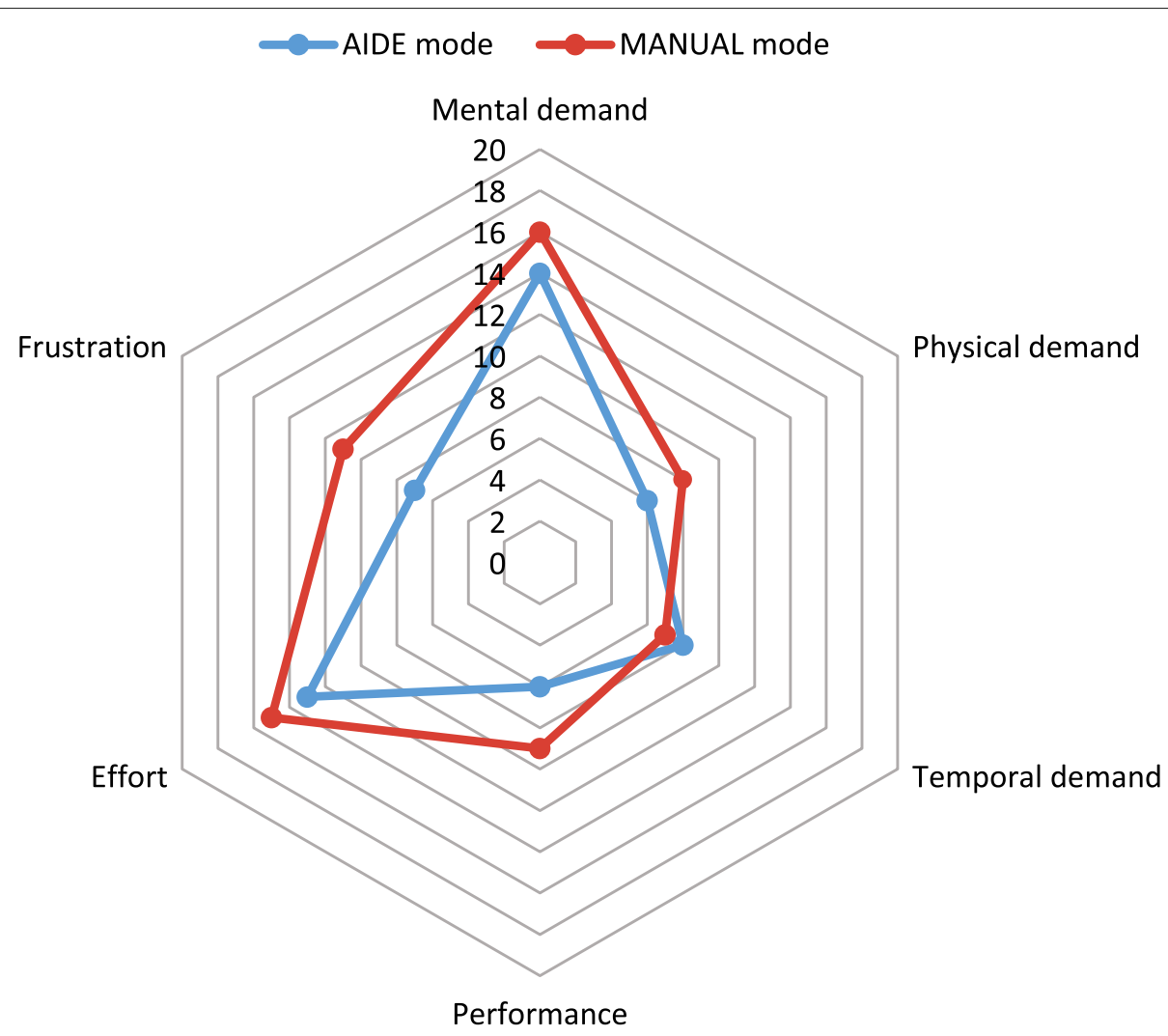

Fig. 5 NASA task load index. The subjects answered the NASA tlx questionnaire after each mode

\section{Conclusion}

The presented ECI allowed the users to perform simulated ADLs with a multimodal control system. The platform was tested in two different scenarios: Manual and AIDE mode. The first one was presented as a simple ECI where the user had to achieve the corresponding ADL. The second mode used a CRF model to predict the user's intention through the environment analysis and identification of the user's behaviors. We conclude that, even the users do not perceive subjective differences between both modes in terms of workload, the AIDE mode helps the user to perform mode ADLs, spending less time per task, showing statistically significant differences with respect to the Manual mode. This effect is caused by the user's intention prediction as the ECI jumps directly to the last abstraction level of the ECI. The environment analysis and the identification of the user's behaviors can be used to predict the user intention and will allow to speed up the ECIs scan opening a new paradigm in the design of these interfaces. Although the developed ECI was tested only in a simulated home environment, it can be easily adapted to a real environment increasing the user independence at home.

\section{Endnotes}

${ }^{1}$ http://aideproject.umh.es/

${ }^{2}$ http://www.thinksmartbox.com

${ }^{3}$ http://www.tobii.com

\section{Abbreviations}

AAC: Augmentative and alternative communication; ADL: Activity of daily living; $\mathrm{BCl}$ : Brain computer interface; CRF: Conditional random field; ECl: Environment control interface; EEG: Electroencephalography; EOG: Electrooculography; HOV: Horizontal oculoversion; MI: Motor Imagery; SMR-ERD: Sensorimotor rhythm event-related desynchronization; SVM: Support vector machine; tlx: task load index

\section{Acknowledgments}

The authors thank the subjects for their participation. In addition, we acknowledge the great support from CEDAR Foundation, Belfast, UK and the Biomedical Research Networking Center (CIBER).

\section{Funding}

This work has been supported by the AIDE project through the grant agreement no. 645322 of the European Commission and by the Ministry of Economy and Competitiveness through the project DPI2015-70415-C2-2-R.

\section{Availability of data and materials}

Data and materials can be made available upon request to the corresponding author. 


\section{Authors' contributions}

ABM, SM, SRS and NGA designed the study. ABM, SE, JAB, LDL and SD collect the data. SD developed the environment control interface. LDL designed the virtual home environment. ABM, SE and JAB interpreted and analyze the data. $A B M, S E$ created the figures. ABM, SE, JAB, LDL and SD wrote the manuscript. $S M, N G A$ and SRS deeply revised the manuscript. ABM, SE, JAB, LDL, SE, MN, SM, SRS and NGA edited the manuscript. All authors read and approved the final manuscript.

\section{Ethics approval and consent to participate}

This study was conducted according to the declaration of Helsinki and had ethical approval by the Office Research Ethics Northern Ireland (June 2016) approval granted for AIDE project (15/NE/0384). All subjects gave written informed consent prior to data collection.

\section{Consent for publication}

Participants gave written informed consent to data treatment in this research study and permission to publish anonymous data and results.

\section{Competing interests}

Sergio Domingo is a software developer at BJ Adaptaciones, the developer of SHX software. Sergio Domingo declares no competing interests. The rest of the authors declare no competing interests.

\section{Publisher's Note}

Springer Nature remains neutral with regard to jurisdictional claims in published maps and institutional affiliations.

\section{Author details}

${ }^{1}$ Miguel Hernández University of Elche, Av. Universidad w/n, Ed. Innova, 03202 Elche, Spain. ${ }^{2}$ BJ Adaptaciones, St Mare de Déu del Coll, 70, 08023 Barcelona, Spain. ${ }^{3}$ The Cedar Foundation, 1 Upper Lisburn Road, BT10 0GW Belfast, UK. ${ }^{4}$ University Hospital of Tuebingen, Applied Neurotechnology Lab, Calwerstr. 14, D-72076 Tübingen, Germany. ${ }^{5}$ Clinical Neurotechnology Laboratory, Neuroscience Research Center (NWFZ), Charité University Medicine Berlin, Charitéplatz 1, 10117 Berlin, Germany.

Received: 25 July 2018 Accepted: 18 November 2018

Published online: 16 January 2019

\section{References}

1. World Health Organization. Neurological Disorders: Public Health Challenges. Geneva: World Health Organization; 2006. http://www.who. int/iris/handle/10665/43605.

2. Winstein CJ, Stein J, Arena R, Bates B, Cherney LR, Cramer SC, Deruyter F, Eng JJ, Fisher B, Harvey RL, et al. Guidelines for adult stroke rehabilitation and recovery: a guideline for healthcare professionals from the american heart association/american stroke association. Stroke. 2016;47(6):98-169.

3. Sellers EW, Vaughan TM, Wolpaw JR. A brain-computer interface for long-term independent home use. Amyotroph Lateral Scler. 2010;11(5): 449-55.

4. Ball MM, Perkins MM, Whittington FJ, Hollingsworth C, King SV, Combs BL. Independence in assisted living. J Aging Stud. 2004;18(4):467-83.

5. Ball MM, Perkins MM, Whittington FJ, Connell BR, Hollingsworth C, King SV, Elrod CL, Combs BL. Managing decline in assisted living: The key to aging in place. J Gerontol B Psychol Sci Soc Sci. 2004;59(4):202-12.

6. Struijk LNA, Egsgaard LL, Lontis R, Gaihede M, Bentsen B. Wireless intraoral tongue control of an assistive robotic arm for individuals with tetraplegia. J Neuroengineering Rehabil. 2017;14(1):110.

7. Brandt Å, Samuelsson K, Töytäri O, Salminen A-L. Activity and participation, quality of life and user satisfaction outcomes of environmental control systems and smart home technology: a systematic review. Disabil Rehabil Assist Technol. 2011;6(3):189-206.

8. Agree EM, Freedman VA, Cornman JC, Wolf DA, Marcotte JE. Reconsidering substitution in long-term care: when does assistive technology take the place of personal care? I Gerontol B Psychol Sci Soc Sci. 2005;60(5):272-80.

9. O'Neill B, Moran K, Gillespie A. Scaffolding rehabilitation behaviour using a voice-mediated assistive technology for cognition. Neuropsychol Rehabil. 2010;20(4):509-27.
10. Lupu RG, Ungureanu F, Siriteanu V. Eye tracking mouse for human computer interaction. In: E-Health and Bioengineering Conference (EHB), 2013. IEEE; 2013. p. 1-4. https://doi.org/10.1109/EHB.2013.6707244.

11. Adjouadi M, Sesin A, Ayala M, Cabrerizo M. Remote eye gaze tracking system as a computer interface for persons with severe motor disability. In: International Conference on Computers for Handicapped Persons. Berlin: Springer; 2004. p. 761-9. https://doi.org/10.1007/978-3-54027817-7_113.

12. Biswas $P$, Langdon $P$. A new input system for disabled users involving eye gaze tracker and scanning interface. J Assist Technol. 2011;5(2):58-66.

13. Miralles F, Vargiu E, Rafael-Palou X, Sola M, Dauwalder S, Guger C, Hintermuller C, Espinosa A, Lowish H, Martin S, Armstrong E, Daly J. Brain?computer interfaces on track to home: Results of the evaluation at disabled end-users? homes and lessons learnt. Front ICT. 2015;2:25. https://doi.org/10.3389/fict.2015.00025.

14. Wolpaw JR, Birbaumer N, McFarland DJ, Pfurtscheller G, Vaughan TM. Brain-computer interfaces for communication and control. Clin Neurophysiol. 2002;113(6):767-91.

15. Cincotti F, Mattia D, Aloise F, Bufalari S, Schalk G, Oriolo G, Cherubini A, Marciani MG, Babiloni F. Non-invasive brain-computer interface system: towards its application as assistive technology. Brain Res Bull. 2008;75(6): 796-803

16. Witkowski M, Gómez C, Opisso E, Medina J, Cortese M, Cempini M, Carrozza MC, Cohen LG, Birbaumer N, Vitiello N. Hybrid eeg/eog-based brain/neural hand exoskeleton restores fully independent daily living activities after quadriplegia. Sci Robot. 2016;1 (1):eaag3296. https://doi. org/10.1126/scirobotics.aag3296.

17. Soekadar SR, Witkowski M, Mellinger J, Ramos A, Birbaumer N, Cohen LG. Erd-based online brain-machine interfaces (bmi) in the context of neurorehabilitation: optimizing bmi learning and performance. IEEE Trans Neural Syst Rehabil Eng: Publ IEEE Eng Med Biol Soc. 2011;19(5):542-9.

18. Lafferty J, McCallum A, Pereira FC. Conditional random fields: Probabilistic models for segmenting and labeling sequence data. In: Proceedings of the Eighteenth International Conference on Machine Learning (ICML '01). San Francisco: Morgan Kaufmann Publishers Inc.; 2001. p. 282-289. http://portal.acm.org/citation.cfm?id=655813.

19. Schalk G, McFarland DJ, Hinterberger T, Birbaumer N, Wolpaw JR. Bci2000: a general-purpose brain-computer interface (bci) system. IEEE Trans Biomed Eng. 2004;51(6):1034-43.

20. Barios JA, Ezquerro S, Bertomeu-Motos A, Nann M, Badesa FJ, Fernandez E, Soekada SR, Garcia-Aracil N. Synchronization of slow cortical rhythms during motor imagery-based brain-machine interface control. Int J Neural Syst. 2018. in press.

21. Rabiner LR. A tutorial on hidden Markov models and selected applications in speech recognition. Proc IEEE. 1989;77(2):257-86.

22. Quinn TJ, Langhorne P, Stott DJ. Barthel index for stroke trials: development, properties, and application. Stroke. 2011;42(4):1146-51.

23. Hart SG, Staveland LE. Development of nasa-tlx (task load index): Results of empirical and theoretical research. In: Advances in Psychology, vol 52. North Holland: Elsevier; 1988. p. 139-83. https://doi.org/10.1016/S01664115(08)62386-9.

\section{Ready to submit your research? Choose BMC and benefit from}

- fast, convenient online submission

- thorough peer review by experienced researchers in your field

- rapid publication on acceptance

- support for research data, including large and complex data types

- gold Open Access which fosters wider collaboration and increased citations

- maximum visibility for your research: over $100 \mathrm{M}$ website views per year

At BMC, research is always in progress.

Learn more biomedcentral.com/submissions 